\title{
EGFR gene status predicts response and survival benefit in a preclinical gastric cancer trial treating patient-derived xenografts with cetuximab
}

\author{
XIAOHONG WANG ${ }^{1 *}$, RUNJIA FU ${ }^{2 *}$, YING HU ${ }^{1}, \mathrm{HONG} \mathrm{DU}^{2}$, SHUANGXI LI ${ }^{3}, \mathrm{ZIYU} \mathrm{LI}^{3}$, \\ YIQIANG LIU ${ }^{4}$, QIXIANG $\mathrm{LI}^{5}$, LIANHAI ZHANG ${ }^{3}$ and JIAFU JI ${ }^{3}$ \\ ${ }^{1}$ Key Laboratory of Carcinogenesis and Translational Research (Ministry of Education), Department of Tissue Bank, \\ ${ }^{2}$ Key Laboratory of Carcinogenesis and Translational Research (Ministry of Education), Gastrointestinal Cancer Translational \\ Research Laboratory, ${ }^{3}$ Key Laboratory of Carcinogenesis and Translational Research (Ministry of Education), \\ Department of Surgery and ${ }^{4}$ Key Laboratory of Carcinogenesis and Translational Research (Ministry of Education), \\ Department of Pathology, Peking University Cancer Hospital and Institute, Beijing 100142; \\ ${ }^{5}$ Crown Bioscience Inc., Light Muller Building, Changping Sector of Zhongguancun Science Park,
} Changping, Beijing 102200, P.R. China

Received December 6, 2016; Accepted June 21, 2017

DOI: $10.3892 /$ or.2017.5907

\begin{abstract}
Cetuximab has been evaluated as a first-line treatment with conflicting results. The aim of the present study was to investigate the relationship between epidermal growth factor receptor (EGFR) status, and response and survival benefit following cetuximab treatment in gastric cancer (GC). Using 20 patient-derived GC xenograft (PDX) models, the mice (10 mice/model) were randomly assigned into two groups. The control group and treatment group were treated with PBS and cetuximab, respectively. The drug response was evaluated by monitoring tumor growth. Survival benefit was evaluated by comparing the survival curves corresponding to the time for the tumors to reach $600 \mathrm{~mm}^{3}$. Our results revealed that the PDX models treated with cetuximab had better survival than that noted for the non-treated group $(\mathrm{P}<0.05)$. The EGFR status was measured by FISH, qPCR, RNAish and immunohistochemistry, respectively. Four cases in the treated group were identified as responsive to cetuximab. EGFR mRNA and protein overexpression were associated with the response to cetuximab $(\mathrm{P}<0.05)$. EGFR amplification, mRNA and protein overexpression were associated with prolonged survival in the cetuximab-treated PDX models. Moreover, in
\end{abstract}

Correspondence to: Professor Lianhai Zhang or Professor Jiafu Ji, Department of Surgery, Peking University Cancer Hospital and Institute, Beijing 100142, P.R. China

E-mail: zlhzlh@hotmail.com

E-mail: jijiafu@hsc.pku.edu.cn

*Contributed equally

Key words: gastric cancer, EGFR, cetuximab, patient-derived xenografts, survival the PDX models with EGFR amplification, mRNA or protein overexpression, cetuximab treatment was associated with a better survival compared with that noted in the untreated group in the PDX models $(\mathrm{P}<0.05)$, while the survival was not statistically different in the other cases $(\mathrm{P}>0.05)$. In conclusion, cetuximab provided survival benefit in the trial. The level of EGFR amplification and overexpression significantly predicted response and survival benefit, particularly the mRNA and protein expression level. A combination of mRNA and protein expression may predict efficacy of cetuximab more efficiently.

\section{Introduction}

Gastric cancer (GC) is an aggressive malignancy and remains a major health issue (1). Chemotherapy improves survival and quality of life compared with the best supportive care, but the median overall survival remains poor (2). There is an urgent need for more effective target agents for treating this disease.

Cetuximab is a recombinant human/mouse chimeric monoclonal antibody against the epidermal growth factor receptor (EGFR). Cetuximab was found to enhance the effect of oxaliplatin in hypoxic GC cell lines (3). Several phase II trials have evaluated cetuximab as a first-line treatment in combination with various chemotherapy regimens (4-6). However, Erbitux in combination with Xeloda and cisplatin in advanced esophago-gastric cancer (EXPAND; NCT00678535) did not significantly increase progression-free survival (PFS) in patients with advanced GC (7).

In vitro and in vivo antitumor activity of cetuximab in human GC cell lines is related to EGFR expression and mutational phenotype $(8,9)$. EGFR mutations have been proven to be the most effective biomarker for the prediction of superior efficacy for EGFR tyrosine kinase inhibitors (TKIs) (10-12). The potential use of EGFR expression as a marker has been widely investigated, with conflicting results (13). Moreover, gene copy 
number gain of EGFR is also a poor prognostic biomarker in GC (8). Unlike HER2 in GC, the predictive value of increased EGFR copy number for tumor response is controversial $(14,15)$. Similarly, the relationship between the level of EGFR amplification and the outcome of EGFR-positive GC treated with first-line chemotherapy with cetuximab remains unclear.

At present, there has been an increase in using experimental models to predict the clinical activity of agents and discover predictive biomarkers. A large collection of patient-derived tumor xenografts (PDXs) reflects the diversity of tumors in patient populations. We established GC PDXs by transplanting surgically removed tumor tissues from patients into immunocompromised BALB/c nude mice via subcutaneous inoculation, to assess drug activity. Moreover, our previous data also suggested that cases with a GC subtype with EGFR amplification and overexpression benefit from cetuximab treatment (16). In the present study, our aim was to determine whether the level of EGFR amplification significantly predicts increased survival and response to therapy in GC treated with cetuximab-based chemotherapy. We investigated the activity of cetuximab in 20 GC-PDX models. After the therapeutic responders and non-responders were identified, the correlation between EGFR amplification, mRNA and protein expression level and tumor response to cetuximab therapy in the GC xenografts were analyzed. Moreover, we also investigated the survival of the GC PDX models in the cetuximab-treated and control groups in regards to the different levels of DNA amplification, mRNA and protein expression.

\section{Materials and methods}

Patient and tumor samples. All of the cases with freshly and surgically removed tumor tissues included in the present study were diagnosed and surgically treated at Peking University Cancer Hospital. The tumor-node-metastasis (TNM) stage was classified according to the 7 th edition of the classification recommended by the American Joint Committee on Cancer (AJCC) (17). All of the tumors were not previously treated with chemotherapy or EGFR inhibitors. This investigation was performed after approval by the Ethics Committee of Peking University Cancer Hospital.

Antitumor activity evaluation. The subcutaneous engraftment of patient tumor fragments into immunocompromised mice was previously described (18). When the tumor volume reached $100-150 \mathrm{~mm}^{3}$, the mice were randomly grouped into two groups of five mice with a similar average tumor volume. Immediately after grouping, the control group was treated with vehicle [phosphate-buffered saline (PBS), weekly intraperitoneal injection or i.p. for 2 weeks], and the treatment groups were injected with cetuximab (weekly i.p. injection for 2 weeks, $50 \mathrm{mg} / \mathrm{kg}$; Merck KGaA, Darmstadt, Germany). The tumor growth was monitored twice weekly, and $\Delta \mathrm{T} / \Delta \mathrm{C}$ value was calculated for assessing tumor response to the treatment $(\Delta \mathrm{T}=$ tumor volume change in the treatment group and $\Delta \mathrm{C}=$ tumor volume change in the control group). The total number of mice used for the xenografts was 200 (10 mice/ model for 20 PDX models). Survival benefit was evaluated by comparing the survival curves corresponding to the time for the tumor to reach $600 \mathrm{~mm}^{3}(19,20)$. All procedures were carried out under sterile conditions at Crown Bioscience SPF facility and conducted in strict accordance with the Guide for the Care and Use of Laboratory Animals of the National Institutes of Health. The protocol was approved by the Committee on the Ethics of Animal Experiments of Crown Bioscience (Crown Bioscience IACUC Committee).

Immunohistochemistry (IHC) staining. Four-micrometer sections from formalin-fixed paraffin-embedded tissues were mounted on poly-L-lysine-coated slides, and then deparaffinized in xylene and rehydrated through alcohol to distilled water. Endogenous peroxidase activity was blocked with $3 \%$ hydrogen peroxide for $15 \mathrm{~min}$ at room temperature. After pressure cooking the slides in $10 \mathrm{mmol} / \mathrm{l}$ EDTA ( $\mathrm{pH}$ 8.0) for $3 \mathrm{~min}$, the sections were incubated overnight at $4^{\circ} \mathrm{C}$ with mouse rabbit anti-human EGFR antibody (Cell Signaling Technology, Inc., Boston, MA, USA) at final dilution of 1:200. Primary antibodies were detected using a two-step EnVision System (Dako, Glostrup, Denmark). Positive and negative immunohistochemistry controls were routinely used. For negative controls, the primary antibody was replaced by non-immune mouse serum to confirm its specificity. Moreover, we used an internal positive control in immunohistochemistry for quality assurance. The test specimens were then independently scored by three investigators in a blinded fashion: score 0 is when there was no specific membrane staining within the tumor, and positive when there was any staining of the tumor cell membrane above the background level. The positive cases were further classified into $1+, 2+$ and $3+$ based on the staining intensity of the membrane.

EGFR gene copy numbers and mRNA expression detection. Quantitative RT-PCR was performed to determine the relative EGFR gene expression level for all of the samples. Extracted mRNA was subjected to amplification using human EGFR-specific primers by TaqMan q-PCR (assay ID, Hs01076078_m1; Applied Biosystems, Foster City, CA, USA). The human GAPDH gene was used as a reference (assay ID, Hs99999905_m1; Applied Biosystems). Each sample was run thrice. Expression of each gene was represented as the ratio of expression of each target gene mRNA to that of GAPDH mRNA. Moreover, Affymetrix HG-U219 array was also performed following a standard protocol (http:// media.affymetrix.com/support/downloads/manuals/3_ivt_ express_kit_manual.pdf) to detect EGFR mRNA expression.

In addition, EGFR gene copy numbers were determined by quantitative PCR. Briefly, the same genomic DNAs were subjected to amplification by TaqMan qPCR. The primers for EGFR (assay ID, Hs04960197_cn) and RNase P as endogenous reference (part no. 4401631) were purchased from Applied Biosystems. The raw data were transferred to CopyCaller software and analyzed.

FISH. Quantitative assessment of EGFR copy number was also investigated by FISH. Dual-color, dual-target FISH assays were carried out using the EGFR Spectrum Orange/CEP7 Spectrum Green Probe (Vysis, Des Plaines, IL, USA). Three-micrometer thick tissue sections were treated with the procedure provided by fluorescence in-situ hybridization (FISH) detection kit (DakoCytomation, Glostrup, Denmark). Briefly, samples were 
placed in pretreatment solution for $30 \mathrm{~min}$ at $96^{\circ} \mathrm{C}$, and digested with pepsin solution for $30 \mathrm{~min}$ at room temperature. Tissue sections, covered with $10-\mu l$ probe solution, were incubated at $75^{\circ} \mathrm{C}$ for $5 \mathrm{~min}$ to co-denature the EGFR and chromosome seven $\alpha$-centromeric (CEP7) probes and allowed to hybridize overnight at $37^{\circ} \mathrm{C}$. Co-denaturation and hybridization were carried out sequentially. Post-hybridization stringency wash was carried out in a water bath at $65^{\circ} \mathrm{C}$ for $10 \mathrm{~min}$. Then, tissue sections were covered with DAPI II (Vysis) for chromatin counterstaining. EGFR was visualized as a red signal with a standard tetramethyl Rhodamine isothiocyanate (TRITC) filter, CEP7 as a green signal with a fluorescein isothiocyanate (FITC) filter, and nuclei as a blue signal with a DAPI filter. Representative images of the samples were acquired and then analyzed.

Two independent observers scored at least 100 non-overlapping interphase nuclei for the number of copies of EGFR and CEP7 by use of predefined scoring guidelines. EGFR status was scored as the number of EGFR signals/nucleus and as the ratio of EGFR signals to CEP7 signals. Amplification was defined as the presence of 2.5 or more signals/nucleus, i.e., EGFR copy number $\geq 2.5$.

RNA in situ hybridization assays in FFPE samples. In situ hybridization (ISH) was performed using QuantiGene ${ }^{\circledR}$ ViewRNA ISH Tissue Assay (Affymetrix, Santa Clara, CA, USA) following the manufacturer's protocol. Briefly, FFPE slides were prepared according to the procedure described. Then, sections were rehydrated and incubated with Proteinase K. Standard probe design software was used to design specific oligonucleotide probe sets for detecting target genes. A no-probe sample was utilized as a negative control per the Affymetrix manual's recommendations. The signal was amplified before incubation with labeled probes and visualized. Hybridized target mRNAs were visualized using confocal fluorescent microscopy, or by bright field microscopy.

The test specimens were then independently scored by three investigators in a blinded fashion: score 0 is when there was no specific staining within the tumor, and positive when there was any staining of the tumor cell membrane above the background level. The positive cases were further classified into $1+, 2+$ and $3+$ based on the staining intensity.

Statistical analysis. In order to investigate correlations between tumor response $(\Delta \mathrm{T} / \Delta \mathrm{C}$ value) and $\mathrm{CN} / \mathrm{mRNA} /$ protein expression, the $\Delta \mathrm{T} / \Delta \mathrm{C}$ value, DNA and mRNA expression value was coded as one for expression levels ranked as at or below the 25 th percentile of the total gene expression, two for levels above the 25th and at or below the 50th percentiles, three for levels above the 50th and at or below the 75th percentiles, and four for levels above the 75th percentile. Spearman rank order correlations were performed to analyze the correlation between tumor response and $\mathrm{CN} / \mathrm{mRNA} /$ protein expression. Hazard ratios from univariate Cox regression analysis were used to determine whether the factor was associated with death. Protective genes were defined as those associated with a hazard ratio for death of $<1$; risk genes were defined as those associated with a hazard ratio for death of $>1$. Kaplan-Meier analysis was used to compare survival of the two groups of PDX models (by $600 \mathrm{~mm}^{3}$ ) with the log-rank test. In all analyses, $\mathrm{P}<0.05$ was considered to indicate statistical significance, and all tests were two-tailed. The
Table I. Correlation between the DNA, RNA and protein expression level of EGFR and cetuximab response.

\begin{tabular}{lcr}
\hline Detection method & Correlation & P-value \\
\hline DNA CN & & \\
qPCR & 0.20 & 0.398 \\
FISH (EGFR/CEP7) & 0.28 & 0.232 \\
FISH (EGFR) & 0.36 & 0.119 \\
mRNA expression & & \\
RNAish & 0.671 & 0.001 \\
U219 intensity & 0.707 & 0.001 \\
qPCR (EGFR/GAPDH) & 0.720 & $<0.001$ \\
Protein & & 0.003 \\
EGFR IHC score & 0.630 & \\
\hline EGFR, epidermal growth factor receptor; IHC, immunohistochemistry. \\
\hline
\end{tabular}

statistical analysis was carried out using SPSS V16.0 software (SPSS, Inc., Chicago, IL, USA).

\section{Results}

EGFR status in the GC xenografts. We established GC-PDX models by surgically transplanting removed tumor tissues from GC patients into immunocompromised BALB/c nude mice subcutaneously. qPCR, FISH (EGFR and EGFR/CEP7), RNAish and immunohistochemical staining were performed to investigate EGFR expression in the $20 \mathrm{GC}$ xenografts. Amplification of EGFR by FISH was determined by EGFR or the EGFR/CEP7 ratio with a range 1.9 to $>15$ or 0.83 to $>2$ (Fig. 1A). The ranges of EGFR CN by qPCR were 1.4-1040.9. The ranges of mRNA by qPCR (EGFR/GAPDH) were 0.00-13.00. RNAish assay showed that 5 of $20 \mathrm{GC}$ xenografts were EGFR positive; one of them showed strong positive (Fig. 1B). For the 20 cases included in the analysis, $7(35 \%)$ were IHC 0, $7(35 \%)$ were $1+, 2(10 \%)$ were $2+$, and $4(20 \%)$ were $3+$ (Fig. 1C).

Correlation between the tumor response and EGFR status in the cetuximab-treated GC xenografts. The tested GC-PDXs fell into two distinct categories according to the drug activity: 4 of $20(20 \%)$ responded with a nearly complete response $(\Delta \mathrm{T} /$ $\Delta \mathrm{C}$ value $<0)$ to cetuximab treatment; 16 of $20(80 \%)$ did not, 1 of 20 had a partial response and 15 had complete resistance $(\Delta \mathrm{T} / \Delta \mathrm{C}$ value $>30 \%)$.

The correlations between tumor response and the level of DNA amplification, mRNA and protein expression in GC xenografts treated with cetuximab were analyzed. Most of the IHC 3+ cases and mRNA overexpression by RNAish and qPCR had a significant positive correlation with the tumor response to cetuximab (Spearman's, RNAish, $\mathrm{P}=0.001$; EGFR/ GAPDH, $\mathrm{P}<0.001$; EGFR IHC score, $\mathrm{P}=0.003$ ), while EGFR $\mathrm{CN}$, either detected by qPCR or FISH (EGFR, EGFR/CEP7), there was no significantly correlation between the EGFR CN and tumor response (EGFR CN detected by qPCR, $\mathrm{P}=0.398$; FISH, $\mathrm{P}=0.119,0.232$, respectively; Table I). 


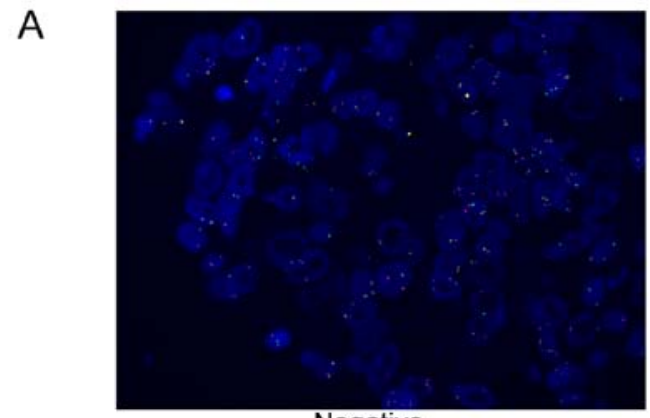

Negative
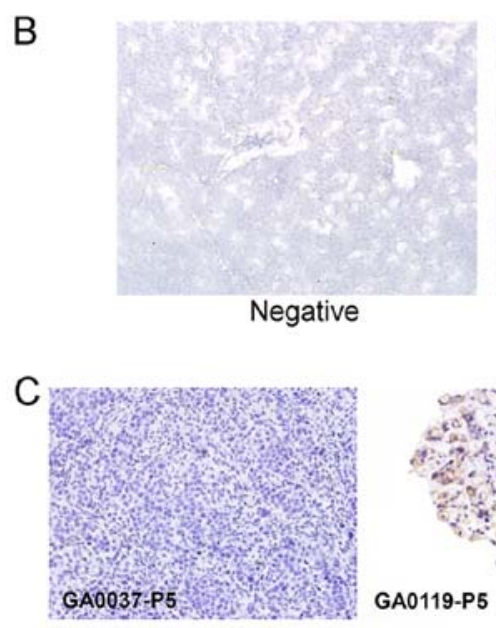

Negative

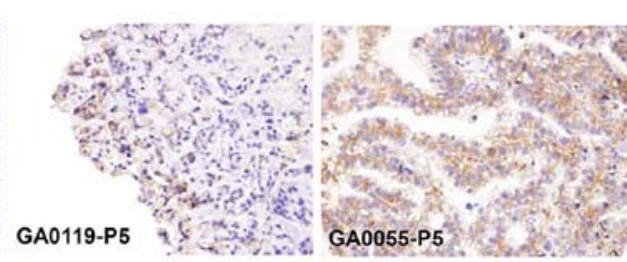

Weak

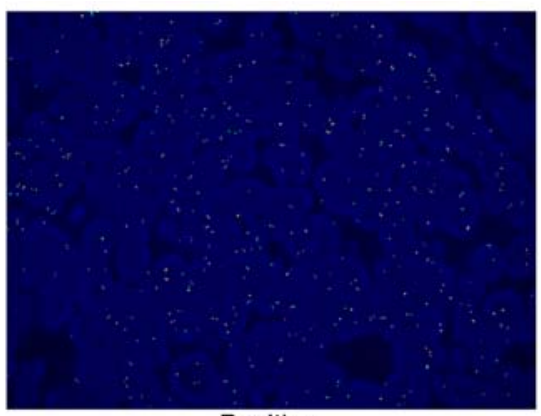

Positive

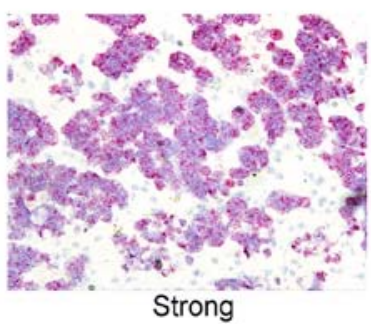

Figure 1. Representative images of (A) FISH, (B) RNAish and (C) IHC.
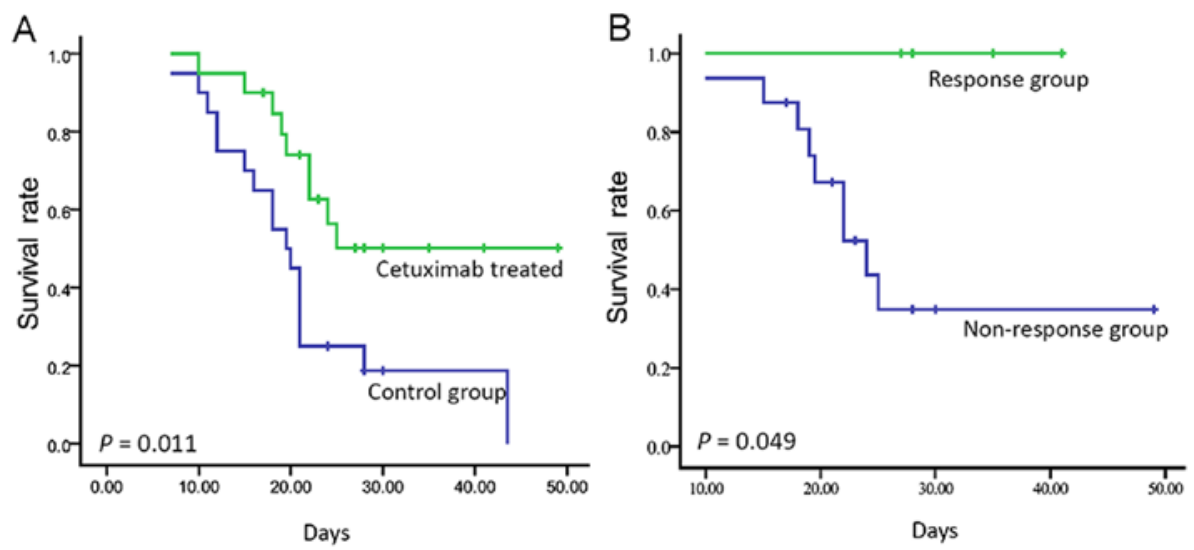

Figure 2. Survival and cetuximab therapy in the PDX models. (A) Survival in the cetuximab-treated and control groups. (B) Survival in the responsive and non-responsive groups.

Survival of the GC PDX models. Survival of the GC PDX were compared between the cetuximab-treated and control group. Our results suggested that the PDX models treated with cetuximab had longer survival compared with that noted in the control cases (median, 19.5 days vs. not reached). This difference was statistically significant (log-rank, $\mathrm{P}=0.011$; Fig. 2A). In the univariate Cox analyses, cetuximab was significantly associated with survival [hazard ratio $0.371 ; 95 \%$ confidence interval (CI), 0.164 to $0.838 ; \mathrm{P}=0.017$ ]. Moreover, the PDX models responsive to cetuximab had longer survival than those in the non-responsive cases (log-rank, $\mathrm{P}=0.049$; Fig. 2B).
Survival and different level of DNA amplification, $m R N A$ and protein expression in cetuximab-treated cases. For the 20 cases, 6 were EGFR $\mathrm{CN} \geq 4$ as detected by qPCR, 14 were EGFR/CEP7 $\geq 1$ as detected by FISH, and 7 were EGFR $\geq 2.5$ by FISH. PDX models treated with cetuximab considered $\mathrm{CN}<4$ had shorter survival than those patients with $\mathrm{CN} \geq 4$ (median, 22.0 days vs. not reached; $\mathrm{P}=0.050$; Fig. 3A). This difference was statistically significant by qPCR, while there was no statistically significant difference detected by FISH (EGFR/FISH, median, 24.0 days vs. not reached, log-rank; $\mathrm{P}=0.221$; Fig. 3B). 

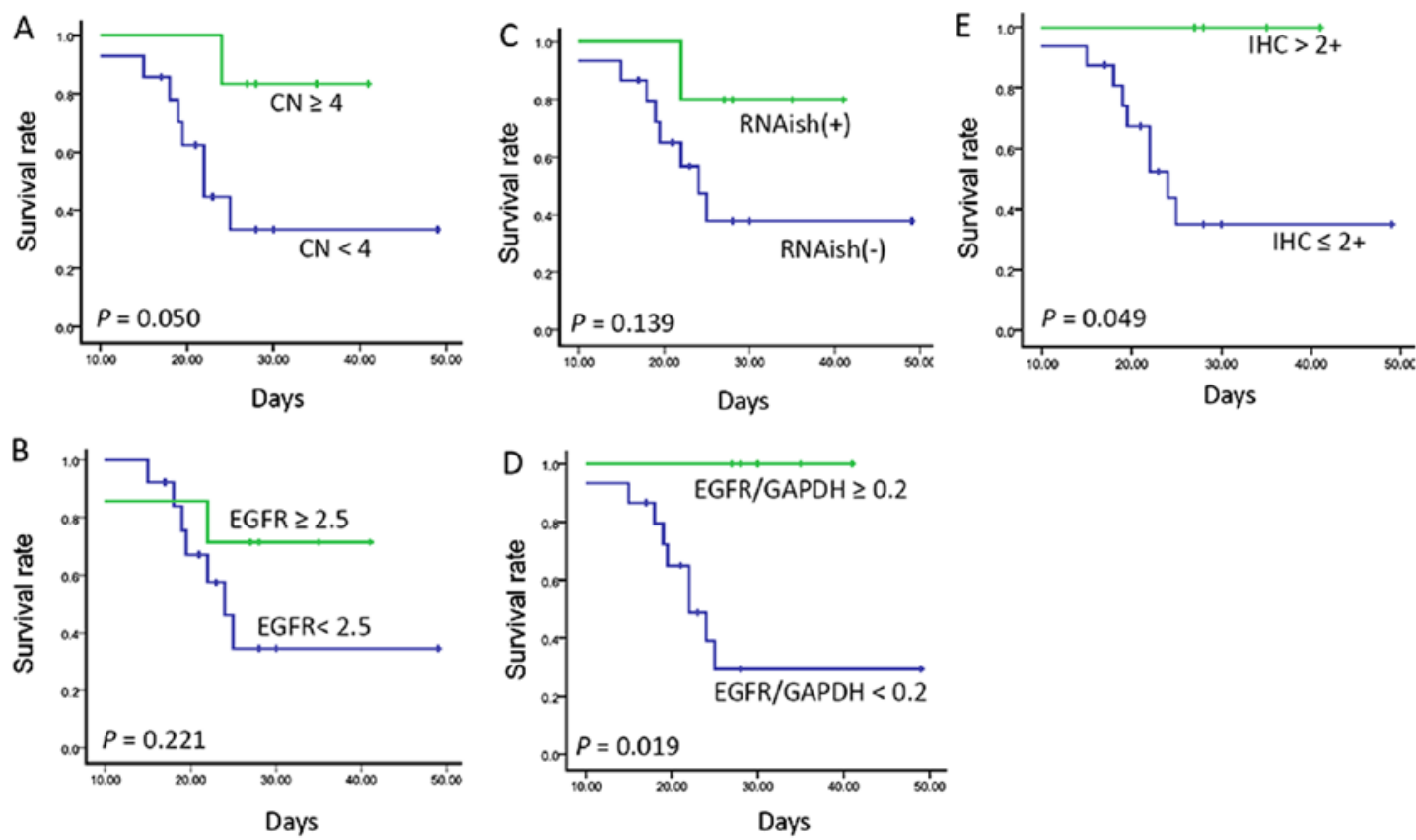

Figure 3. The survival and different level of DNA amplification, mRNA and protein expression in the cetuximab treatment group. (A) DNA (qPCR). (B) FISH (EGFR). (C) RNA (RNAish). (D) RNA (qPCR). (E) Protein (IHC).
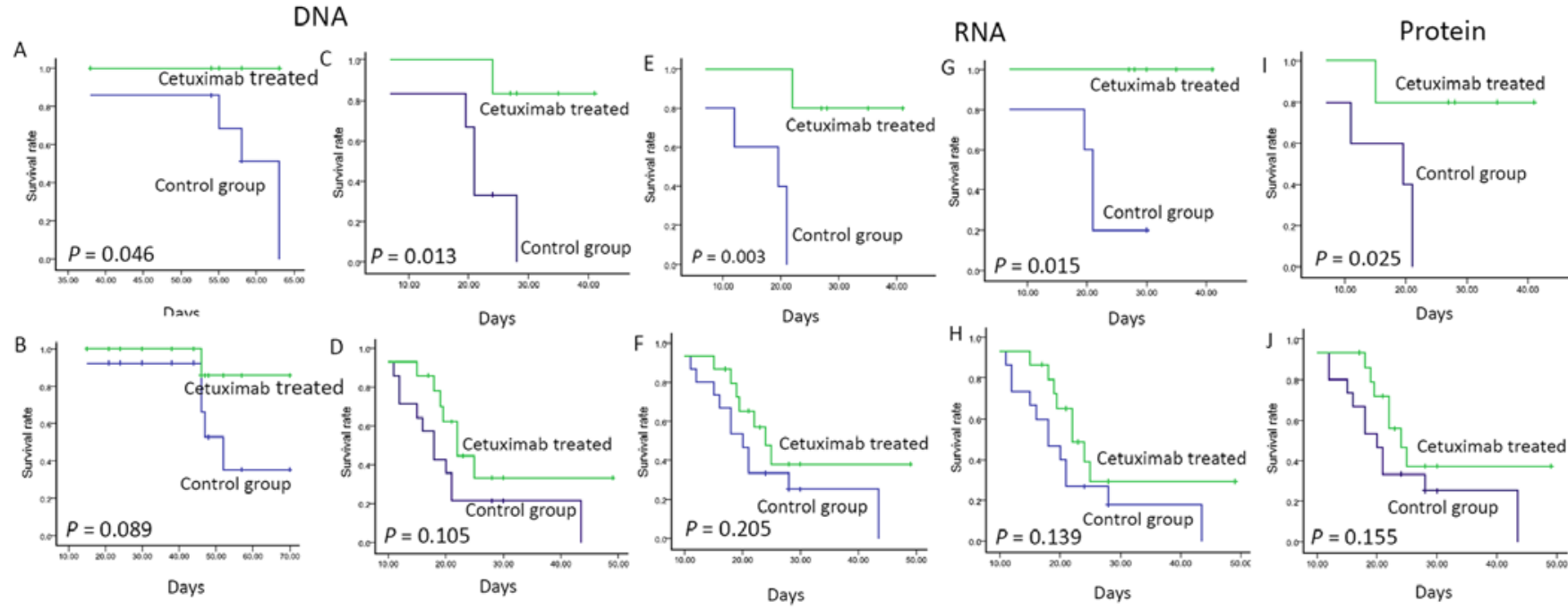

Figure 4. Survival rate of the cetuximab treatment and control groups with different CN, mRNA expression and protein expression. (A) CN (FISH, EGFR $\geq 2.5$ ). (B) CN (FISH, EGFR <2.5). (C) CN (qPCR, $\geq 4$ ). (D) CN (qPCR, <4). (E) RNAish (+). (F) RNAish (-). (G) mRNA (qPCR, $\geq 0.2)$. (H) mRNA (qPCR, <0.2). (I) Protein ( $\mathrm{IHC}>2+)$. (J) Protein ( $\mathrm{IHC} \leq 2+$ ).

Then, we evaluated EGFR mRNA and protein expression level and the survival following treatment with cetuximab. Of these, 5 cases had EGFR mRNA overexpression as detected by RNAish or qPCR (EGFR/GAPDH). Their survival was longer than that of those with a lower mean (RNAish, median, 24.0 days vs. not reached, log-rank; $\mathrm{P}=0.139$; qPCR, log-rank; $\mathrm{P}=0.019$, respectively; Fig. $3 \mathrm{C}$ and $\mathrm{D})$. The EGFR protein expression of 4 patients was IHC $3+$; survival of these cases was better than that of those with a lower expression (log-rank; $\mathrm{P}=0.049$; Fig. 3E).

Survival and cetuximab therapy in different EGFR copy number subgroup. Amplification: for both EGFR/CEP7 ratio value, one was used as the optimal cut-off value that discriminated between the GC PDX model with longer survival and those who more probably may respond to cetuximab-based therapy. As illustrated in Fig. 4A in the cases with EGFR $\geq 2.5$, the median survival of the cetuximab-treated group was significantly longer than that of the non-treated group (logrank; $\mathrm{P}=0.046$ ). Similarly, cases with $\mathrm{EFGR} \mathrm{CN}>4$ by qPCR showed an increased and statistically significant benefit in survival (median, 21.0 vs. not reached; log-rank, $\mathrm{P}=0.013$; Fig. 4C). However, either in the group with EGFR $<2.5$ by FISH or EFGR $\mathrm{CN}<4$ by qPCR, the survival rate was not statistically significant between the cetuximab-treated and control group (EGFR/FISH, median, 52.0 days vs. not 
reached, log-rank; P=0.089; EGFR CN/Q-PCR median, 18.0 vs. 22.0 days, log-rank; $\mathrm{P}=0.105$, respectively; Fig. $4 \mathrm{~B}$ and $\mathrm{D}$ ).

Survival and cetuximab therapy in the different EGFR $m R N A$ expression level subgroups. The mRNA expression of EGFR was detected by RNAish and EGFR/GAPDH. In the EGFR expression-positive cases (detected by RNAish), the median survival of the cetuximab-treated group was significantly longer than that of the non-treated group (median, 19.5 days vs. not reached; log-rank, $\mathrm{P}=0.003$; Fig. $4 \mathrm{E}$ ), while in the RNAish-negative cases, the survival rate did not differ significantly (median survival, 20.0 vs. 24.0 days; $\mathrm{P}=0.205$; Fig. 4F).

Similarly, in the EGFR/GAPDH $\geq 0.2$ group, those in the cetuximab-treated group showed a longer survival rate than those of the control cases (median survival; $\mathrm{P}=0.015$; Fig. 4G), while in the EGFR/GAPDH $<0.2$ group, the survival rate did not significantly differ in the cetuximab-treated and control cases (median survival, 18.0 vs. 22 days; $\mathrm{P}=0.139$; Fig. $4 \mathrm{H}$ ).

Survival and cetuximab therapy in the different EGFR protein expression level subgroup. Moreover, the survival rate and cetuximab therapy in the GC PDX models with different levels of EGFR protein expression was also investigated. A score of $2+$ was used as the optimal EGFR IHC score cut-off value. In the EGFR protein overexpression cases, the median survival rate of the cetuximab-treated group was significantly longer than that of the non-treated group (median, 19.5 days vs. not reached; log-rank, $\mathrm{P}=0.025$; Fig. 4I), while in the group with EGFR expression IHC score $<2$ cases, the survival rate did not differ significantly (median, 20.0 vs. 24.0 days; log-rank, $\mathrm{P}=0.155$; Fig. 4J).

Combined detection of DNA amplification, $m R N A$ and protein overexpression and the survival rate and tumor response to cetuximab therapy. DNA amplification, mRNA and protein expression are commonly detected by FISH, RNAish and IHC in clinical testing. Combined detection is more meaningful for drug use. We found that 4 of the patients were positive for combined detection, which was consistent with the nearly complete response cases; the others were negative. In the combined positive cases, the median survival rate of the cetuximab-treated group was significantly longer than the negative cases (log-rank, $\mathrm{P}=0.049$; data not shown).

\section{Discussion}

The potential use of EGFR expression as a marker has been widely investigated, with conflicting results. In the present study, our aim was to determine whether the level of EGFR gene amplification, mRNA and protein level could significantly predict some benefit in the survival and response to cetuximab in GC xenografts. EGFR DNA amplification was detected by EGFR/CEP7 ratio and qPCR, mRNA expression was detected by qPCR, RNAish, EGFRIU219 and protein overexpression was detected by IHC. EGFR protein and mRNA expression levels allowed us to identify and discriminate those patients with prolonged survival. We showed, for the first time to the best of our knowledge, how the level of EGFR gene amplification, mRNA and protein expression may be used as a predictive factor for response to cetuximab-based treatment and also for survival benefit in this subset of GC patients through PDX models.

Benefit from the addition of an anti-EGFR agent to chemotherapy could not be confirmed in the phase III trials comparing chemotherapy with and without anti-EGFR agent, including the randomized EXPAND and REAL3 trials. An important point is that neither of these trials selected patients based upon biomarkers $(7,21)$. Various authors have suggested that both IHC and FISH should be used to determine the HER2 target chemotherapy status in GC (22). However, EGFR status evaluation method mostly focused on the gene mutations of downstream genes, such as KRAS and BRAF. EGFR protein expression was found to be associated with the survival rate in GC (23). In the present study, we tested the RAS mutation status. There was no RAS mutation in these tumor samples. Our results showed that cases with high EGFR mRNA expression and immunohistochemistry score were more prone to response to cetuximab. EGFR mRNA and protein overexpression were associated with the survival rate in the cetuximab-treated PDX models. Moreover, in the PDX models derived from mRNA or protein overexpression cases, the survival rate of the cetuximab-treated PDX models was significantly longer than that noted in the control group, while the survival was not statistically different in the other cases. The potential use of EGFR expression as a marker has been widely investigated in other cancers, such as NSCLC, with conflicting results. Previous report suggested that EGFR overexpression is associated with improved response, longer time to progression and improved survival in NSCLC patients treated with gefitinib (24). Biomarker analysis of the BR.21 study showed that survival among patients with high EGFR expression was longer in the erlotinib arm vs. the placebo arm, whereas a limited advantage of erlotinib treatment was noted in patients with EGFR IHC-negative tumors (25). This result is similar with ours detected in GC (25).

Gene copy number gain of EGFR is a poor prognostic biomarker in GC (8). At present, there are few studies that have focused on the association of EGFR gene copy number and efficacy of cetuximab chemotherapy, including evaluation of EGFR gene copy number as a predictive biomarker for the efficacy of cetuximab in combination with chemotherapy in the first-line treatment of recurrent and/or metastatic squamous cell carcinoma of the head and neck: EXTREME study (15). The association of EGFR and tumor response to cetuximab should be further studied. In the present study, similar to Her2 amplification (2), we also found that EGFR gene copy number was a predictive biomarker for the efficacy of cetuximab in a GC PDX model, similar with the results in previous studies (26). Compared to EGFR DNA amplification, mRNA and protein overexpression may be more accurate to determine the tumor response to cetuximab. This may be due to other regulatory methods of EGFR expression, such as epigenetics.

In summary, the level of EGFR gene amplification significantly predicted the sensitivity to therapy and the survival rate in GC cases treated with cetuximab-based chemotherapy. This result supports the proposed combined use of IHC and in situ hybridization in this setting. The study of the level of DNA amplification, mRNA and protein expression as a continuous biomarker is arguably a more rational approach for selecting patients more likely to benefit from anti-EGFR-based therapies. 


\section{Acknowledgements}

The present study was partially supported by the National Natural Science Foundation of China (no. 81101879), the NationalKeyTechnology R\&DProgram(2011ZX09307-001-05 and 2014AA020603), and the Beijing Municipal Science and Technology Commission (Z121100007512010).

\section{References}

1. Jemal A, Bray F, Center MM, Ferlay J, Ward E and Forman D: Global cancer statistics. CA Cancer J Clin 61: 69-90, 2011.

2. Gomez-Martin C, Plaza JC, Pazo-Cid R, Salud A, Pons F, Fonseca P, Leon A, Alsina M, Visa L, Rivera F, et al: Level of $H E R 2$ gene amplification predicts response and overall survival in HER2-positive advanced gastric cancer treated with trastuzumab. J Clin Oncol 31: 4445-4452, 2013.

3. Luo HY, Wei W, Shi YX, Chen XQ, Li YH, Wang F, Qiu MZ, Li FH, Yan SL, Zeng MS, et al: Cetuximab enhances the effect of oxaliplatin on hypoxic gastric cancer cell lines. Oncol Rep 23: $1735-1745,2010$

4. Pinto C, Di Fabio F, Siena S, Cascinu S, Rojas Llimpe FL, Ceccarelli C, Mutri V, Giannetta L, Giaquinta S, Funaioli C, et al: Phase II study of cetuximab in combination with FOLFIRI in patients with untreated advanced gastric or gastroesophageal junction adenocarcinoma (FOLCETUX study). Ann Oncol 18 : 510-517, 2007.

5. Han SW, Oh DY, Im SA, Park SR, Lee KW, Song HS, Lee NS, Lee KH, Choi IS, Lee MH, et al: Phase II study and biomarker analysis of cetuximab combined with modified FOLFOX6 in advanced gastric cancer. Br J Cancer 100: 298-304, 2009.

6. Kim C, Lee JL, Ryu MH, Chang HM, Kim TW,Lim HY, Kang HJ, Park YS, Ryoo BY and Kang YK: A prospective phase II study of cetuximab in combination with XELOX (capecitabine and oxaliplatin) in patients with metastatic and/or recurrent advanced gastric cancer. Invest New Drugs 29: 366-373, 2011.

7. Lordick F, Kang YK, Chung HC, Salman P, Oh SC, Bodoky G, Kurteva G, Volovat C, Moiseyenko VM, Gorbunova V, et al; Arbeitsgemeinschaft Internistische Onkologie and EXPAND Investigators: Capecitabine and cisplatin with or without cetuximab for patients with previously untreated advanced gastric cancer (EXPAND): A randomised, open-label phase 3 trial. Lancet Oncol 14: 490-499, 2013.

8. Higaki E, Kuwata T, Nagatsuma AK, Nishida Y, Kinoshita T, Aizawa M5, Nitta H, Nagino M and Ochiai A: Gene copy number gain of $E G F R$ is a poor prognostic biomarker in gastric cancer: Evaluation of 855 patients with bright-field dual in situ hybridization (DISH) method. Gastric Cancer 19: 63-73, 2016.

9. Hotz B, Keilholz U, Fusi A, Buhr HJ and Hotz HG: In vitro and in vivo antitumor activity of cetuximab in human gastric cancer cell lines in relation to epidermal growth factor receptor (EGFR) expression and mutational phenotype. Gastric Cancer 15: 252-264, 2012

10. Zhou C, Wu YL, Chen G, Feng J, Liu XQ, Wang C, Zhang S, Wang J, Zhou S, Ren S, et al: Erlotinib versus chemotherapy as first-line treatment for patients with advanced EGFR mutationpositive non-small-cell lung cancer (OPTIMAL, CTONG-0802): A multicentre, open-label, randomised, phase 3 study. Lancet Oncol 12: 735-742, 2011.

11. Maemondo M, Inoue A, Kobayashi K, Sugawara S, Oizumi S, Isobe H, Gemma A, Harada M, Yoshizawa H, Kinoshita I, et al; North-East Japan Study Group: Gefitinib or chemotherapy for non-small-cell lung cancer with mutated EGFR. N Engl J Med 362: 2380-2388, 2010.

12. Mitsudomi T, Morita S, Yatabe Y, Negoro S, Okamoto I, Tsurutani J, Seto T, Satouchi M, Tada H, Hirashima T, et al; West Japan Oncology Group: Gefitinib versus cisplatin plus docetaxel in patients with non-small-cell lung cancer harbouring mutations of the epidermal growth factor receptor (WJTOG3405): An open label, randomised phase 3 trial. Lancet Oncol 11: 121-128, 2010.
13. Mazières J, Brugger W, Cappuzzo F, Middel P, Frosch A, Bara I, Klingelschmitt $G$ and Klughammer B: Evaluation of EGFR protein expression by immunohistochemistry using $\mathrm{H}$-score and the magnification rule: Re-analysis of the SATURN study. Lung Cancer 82: 231-237, 2013.

14. Dahabreh IJ, Linardou H, Kosmidis P, Bafaloukos D and Murray S: EGFR gene copy number as a predictive biomarker for patients receiving tyrosine kinase inhibitor treatment: A systematic review and meta-analysis in non-small-cell lung cancer. Ann Oncol 22: 545-552, 2011.

15. Licitra L, Mesia R, Rivera F, Remenár E, Hitt R, Erfán J, Rottey S, Kawecki A, Zabolotnyy D, Benasso M, et al: Evaluation of $E G F R$ gene copy number as a predictive biomarker for the efficacy of cetuximab in combination with chemotherapy in the first-line treatment of recurrent and/or metastatic squamous cell carcinoma of the head and neck: EXTREME study. Ann Oncol 22: 1078-1087, 2011.

16. Zhang L, Yang J, Cai J, Song X, Deng J, Huang X, Chen D, Yang M, Wery JP, Li S, et al: A subset of gastric cancers with EGFR amplification and overexpression respond to cetuximab therapy. Sci Rep 3: 2992, 2013.

17. Washington $\mathrm{K}: 7$ th edition of the AJCC cancer staging manual: Stomach. Ann Surg Oncol 17: 3077-3079, 2010.

18. Yang M, Shan B, Li Q, Song X, Cai J, Deng J, Zhang L, Du Z, Lu J, Chen T, et al: Overcoming erlotinib resistance with tailored treatment regimen in patient-derived xenografts from naïve Asian NSCLC patients. Int J Cancer 132: E74-84, 2013.

19. Julien S, Merino-Trigo A, Lacroix L, Pocard M, Goéré D, Mariani P, Landron S, Bigot L, Nemati F, Dartigues P, et al: Characterization of a large panel of patient-derived tumor xenografts representing the clinical heterogeneity of human colorectal cancer. Clin Cancer Res 18: 5314-5328, 2012.

20. Schleich N, Po C, Jacobs D, Ucakar B, Gallez B, Danhier F and Préat V: Comparison of active, passive and magnetic targeting to tumors of multifunctional paclitaxel/SPIO-loaded nanoparticles for tumor imaging and therapy. J Control Release 194: 82-91, 2014.

21. Waddell T, Chau I, Cunningham D, Gonzalez D, Okines AF, Okines C, Wotherspoon A, Saffery C, Middleton G, Wadsley J, et al: Epirubicin, oxaliplatin, and capecitabine with or without panitumumab for patients with previously untreated advanced oesophagogastric cancer (REAL3): A randomised, open-label phase 3 trial. Lancet Oncol 14: 481-489, 2013.

22. Fox SB, Kumarasinghe MP, Armes JE, Bilous M, Cummings MC, Farshid G, Fitzpatrick N, Francis GD, McCloud PI, Raymond W, et al: Gastric HER2 Testing Study (GaTHER): An evaluation of gastric/gastroesophageal junction cancer testing accuracy in Australia. Am J Surg Pathol 36: 577-582, 2012.

23. Tang D, Liu CY, Shen D, Fan S, Su X, Ye P, Gavine PR and Yin X: Assessment and prognostic analysis of EGFR, HER2, and HER3 protein expression in surgically resected gastric adenocarcinomas. Onco Targets Ther 8: 7-14, 2014.

24. Cappuzzo F, Hirsch FR, Rossi E, Bartolini S, Ceresoli GL, Bemis L, Haney J, Witta S, Danenberg K, Domenichini I, et al: Epidermal growth factor receptor gene and protein and gefitinib sensitivity in non-small-cell lung cancer. J Natl Cancer Inst 97: 643-655, 2005.

25. Tsao MS, Sakurada A, Cutz JC, Zhu CQ, Kamel-Reid S, Squire J, Lorimer I, Zhang T, Liu N, Daneshmand M, et al: Erlotinib in lung cancer - molecular and clinical predictors of outcome. $\mathrm{N}$ Engl J Med 353: 133-144, 2005.

26. Peled N, Yoshida K, Wynes MW and Hirsch FR: Predictive and prognostic markers for epidermal growth factor receptor inhibitor therapy in non-small cell lung cancer. Ther Adv Med Oncol 1: 137-144, 2009. 